\title{
Comprehensive Understanding of Multiple Resonance Thermally Activated Delayed Fluorescence via Quantum Chemistry Calculations
}

\section{Katsuyuki Shizu}

Kyoto University

Hironori Kaji ( $\sim$ kaji@scl.kyoto-u.ac.jp )

Kyoto University https://orcid.org/0000-0002-5111-3852

\section{Article}

Keywords:

Posted Date: February 15th, 2022

DOI: https://doi.org/10.21203/rs.3.rs-1360800/v1

License: (c) (1) This work is licensed under a Creative Commons Attribution 4.0 International License.

Read Full License

Version of Record: A version of this preprint was published at Communications Chemistry on April 14th, 2022. See the published version at https://doi.org/10.1038/s42004-022-00668-6. 


\title{
Comprehensive Understanding of Multiple
}

\author{
Resonance Thermally Activated Delayed
}

\section{Fluorescence via Quantum Chemistry Calculations}

Katsuyuki Shizu ${ }^{l}$ and Hironori Kaji ${ }^{1 *}$

Email:kaji@scl.kyoto-u.ac.jp

${ }^{1}$ Institute for Chemical Research, Kyoto University, Uji, Kyoto 611-0011, Japan 


\begin{abstract}
Molecules that exhibit multiple resonance (MR) type thermally activated delayed fluorescence (TADF) are highly efficient electroluminescent materials with narrow emission spectra. Despite their importance in various applications, the emission mechanism is still controversial. Here, a comprehensive understanding of the mechanism for a representative MR-TADF molecule (5,9diphenyl-5,9-diaza-13b-boranaphtho[3,2,1-de]anthracene, DABNA-1) is presented. Using the equation-of-motion coupled-cluster singles and doubles method and Fermi's golden rule, we quantitatively reproduced all rate constants relevant to the emission mechanism; prompt and delayed fluorescence, internal conversion (IC), intersystem crossing, and reverse intersystem crossing (RISC). In addition, the photoluminescence quantum yield and its prompt and delayed contributions were quantified by calculating the population kinetics of excited states and the transient photoluminescence decay curve. The calculations also revealed that TADF occurred via a stepwise process of thermally activated IC from electronically excited $\mathrm{T}_{1}$ to the second-lowest triplet state, $\mathrm{T}_{2}$, RISC from $\mathrm{T}_{2}$ to $\mathrm{S}_{1}$, and fluorescence from $\mathrm{S}_{1}$.
\end{abstract}




\section{Introduction}

Since highly efficient thermally activated delayed fluorescence (TADF) was first used in organic light-emitting diodes (OLEDs), ${ }^{1}$ many TADF molecules containing various electron donor and acceptor groups have been developed. ${ }^{2-9}$ An important factor for TADF efficiency is the energy difference between the lowest triplet state $\left(\mathrm{T}_{1}\right)$ and the lowest excited singlet state $\left(\mathrm{S}_{1}\right)$, $\Delta E\left(\mathrm{~T}_{1} \rightarrow \mathrm{S}_{1}\right) ;$ a small $\Delta E\left(\mathrm{~T}_{1} \rightarrow \mathrm{S}_{1}\right)(<200 \mathrm{meV})$ is required to induce $\mathrm{T}_{1} \rightarrow \mathrm{S}_{1}$ reverse intersystem crossing (RISC). Another important factor is a large transition dipole moment between $\mathrm{S}_{1}$ and ground-state $\mathrm{S}_{0}$ to accelerate the rate of $\mathrm{S}_{1} \rightarrow \mathrm{S}_{0}$ fluorescence. Experimentally, efficient RISC and high photoluminescence quantum yields (PLQYs) have been simultaneously realized by combining suitable donor and acceptor units that control the spatial overlap between the highestoccupied molecular orbitals (HOMOs) and the lowest-unoccupied molecular orbitals (LUMOs). ${ }^{1-}$

${ }^{9}$ It is important to note that the fluorescence spectra were broadened because of the chargetransfer character of $\mathrm{S}_{1}$, which was a significant drawback for OLED applications in displays.

In 2016, Hatakeyama et al. developed a new class of TADF molecules. ${ }^{10}$ Using a triphenylboron core possessing two nitrogen atoms (5,9-diphenyl-5,9-diaza-13bboranaphtho[3,2,1-de]anthracene, DABNA-1, Fig. 1a), they observed a HOMO-LUMO separation without a conventional donor-acceptor structure, providing efficient TADF with a narrow emission spectrum. The HOMO-LUMO separation resulted from a multiple resonance (MR) effect, that is an opposite resonance effect induced by the boron and nitrogen atoms. MRTADF molecules emitting blue-to-red fluorescence have been reported. ${ }^{11-39}$

Several theoretical studies have attempted to reveal the TADF mechanism in MR molecules. Northey et al. investigated an intersystem-crossing (ISC) mechanism in DABNA-1 
using quantum dynamics and time-dependent density-functional theory (TD-DFT) at the PBE0/6-31G(d) level. ${ }^{40}$ There was only a $0.02 \mathrm{eV}$ energy difference between $\mathrm{T}_{2}$ and $\mathrm{S}_{1}$, $\Delta E\left(\mathrm{~T}_{2} \rightarrow \mathrm{S}_{1}\right)$. However, $\Delta E\left(\mathrm{~T}_{1} \rightarrow \mathrm{S}_{1}\right)$ and $\Delta E\left(\mathrm{~T}_{1} \rightarrow \mathrm{T}_{2}\right)$ were large, $0.59 \mathrm{eV}$ and $0.61 \mathrm{eV}$, respectively, which could not explain efficient RISC from $\mathrm{T}_{1}$ to $\mathrm{S}_{1}$. Gao et al. examined the density-functional dependence of $\Delta E\left(\mathrm{~T}_{1} \rightarrow \mathrm{S}_{1}\right)$ within the framework of TD-DFT. ${ }^{41}$ The MPWK1CIS functional reproduced the experimental $\Delta E\left(\mathrm{~T}_{1} \rightarrow \mathrm{S}_{1}\right),{ }^{41}$ as reviewed by Suresh $e t$ $a l .{ }^{21}$ However, the RISC rate constant $\left(k_{\mathrm{RISC}}\right)$ was not calculated and the TADF mechanism was unclear. Also, they considered TADF in terms of direct (one-step) $\mathrm{T}_{1} \rightarrow \mathrm{S}_{1}$ RISC, which differed from the work of Northey et al. ${ }^{40}$ Pershin et al. reported that TD-DFT methods overestimated $\Delta E\left(\mathrm{~T}_{1} \rightarrow \mathrm{S}_{1}\right)$ for MR molecules, and that the spin-component-scaling second-order approximate coupled-cluster (SCS-CC2) method outperformed TD-DFT for predicting $\Delta E\left(\mathrm{~T}_{1} \rightarrow \mathrm{S}_{1}\right){ }^{42,43}$ The partial inclusion of double excitations within the SCS-CC2 method was responsible for the improved accuracy in predicting $\Delta E\left(\mathrm{~T}_{1} \rightarrow \mathrm{S}_{1}\right)$. The SCS-CC2 calculation of DABNA-1-based molecules revealed the relationship between its molecular structure and electronic properties, $\Delta E\left(\mathrm{~T}_{1} \rightarrow \mathrm{S}_{1}\right)$, and the fluorescence rate constant. However, the TADF mechanism was still unclear because the $k_{\mathrm{RISC}}$ was not calculated. Lin et al. calculated rate constants for fluorescence $\left(k_{\mathrm{F}}\right)$, ISC ( $\left.k_{\mathrm{ISC}}\right), k_{\mathrm{RISC}}$, and internal conversion (IC) $\left(k_{\mathrm{IC}}\right)$ for DABNA- $1 .{ }^{44}$ However, the calculated $k_{\text {RISC }}$ was $6.7 \times 10^{2}\left(\mathrm{~s}^{-1}\right)$, which was much less than the experimental value of $1.0 \times 10^{4}$ $\mathrm{s}^{-1} \cdot{ }^{10}$ The calculated $k_{\mathrm{ISC}}$ of $1.4 \times 10^{4}\left(\mathrm{~s}^{-1}\right)$ was two orders of magnitude less than the experimental value of $4.5 \times 10^{6} \mathrm{~s}^{-1} \cdot{ }^{10} \mathrm{In}$ addition, the calculated non-radiative decay, $k_{\mathrm{IC}}\left(\mathrm{S}_{1} \rightarrow \mathrm{S}_{0}\right)$, was greater than the $k_{\mathrm{F}}\left(\mathrm{S}_{1} \rightarrow \mathrm{S}_{0}\right)$, suggesting that the PLQY of DABNA-1 was less than $50 \%$, in contrast with the $88 \%$ experimental value. ${ }^{10}$ All these previous studies ${ }^{40-44}$ only partially explained the photophysical properties of MR-TADF mainly because crucial RISC was not elucidated. 
Here, we report a comprehensive understanding of the TADF mechanism using the representative MR molecule, DABNA-1. So far, $\Delta E\left(\mathrm{~T}_{1} \rightarrow \mathrm{S}_{1}\right)$ (and spin-orbit coupling (SOC) in some cases) and oscillator strength have been considered to understand the TADF mechanism and to design TADF molecules. However, these are not sufficient for the above aim; quantifications of all types of rate constants and those of energy levels including higher-lying states relevant to the emission process are required for the complete understanding of the emission mechanism. Recently, we reported rate-constant predictions enabled by a proposed cost-effective calculation method based on Fermi's golden rule. ${ }^{45}$ Using the method, we calculated $k_{\mathrm{IC}}, k_{\mathrm{ISC}}, k_{\mathrm{RISC}}, k_{\mathrm{F}}$, and the rate constant for phosphorescence ( $\left.k_{\mathrm{Phos}}\right)$, of benzophenone as an example. The calculated rate constants agreed well with experimental values. Here, we applied the same method to DABNA-1 and determined all relevant rate constants. We also determined the lifetime of the delayed component in transient PL (trPL), the rate constant of TADF $\left(k_{\mathrm{TADF}}\right)$ and the PLQY, by calculating the population kinetics of the excited states and the trPL decay curve. The calculations also indicated that, after photoexcitation, $\mathrm{T}_{1}$ was first generated via $\mathrm{S}_{1} \rightarrow \mathrm{T}_{2}$ ISC and $\mathrm{T}_{2} \rightarrow \mathrm{T}_{1}$ IC. Then, triplet-to-singlet conversion occurred via thermally activated $\mathrm{T}_{1} \rightarrow \mathrm{T}_{2}$ IC and $\mathrm{T}_{2} \rightarrow \mathrm{S}_{1}$ RISC.

\section{Results}

Calculation of matrix elements between vibronic states. Geometry optimization and frequency analysis of $\mathrm{S}_{0}$ for DABNA-1 were performed using the TPSSh $/ 6-31+\mathrm{G}(\mathrm{d})$ method, whereas those of $S_{1}, T_{1}$, and $T_{2}$ were performed using the TD-TPSSh $/ 6-31+G(d)$ method. The polarizable continuum model (PCM) of a $\mathrm{CH}_{2} \mathrm{Cl}_{2}$ solvent was used to consider the effects on 
vibronic states. We used the functional and the PCM conditions for ground- and excited-state geometry optimizations because Gao et al. reported that the TPSSh/6-31+G(d)-PCM model reproduced experimental DABNA-1 emission and absorption wavelengths in $\mathrm{CH}_{2} \mathrm{Cl}_{2}{ }^{41}$ Geometry optimization and frequency analysis were performed with the Gaussian 16 program package. ${ }^{46}$ For MR molecules, the equation-of-motion coupled-cluster singles and doubles (EOM-CCSD) method and algebraic diagrammatic construction of the second order, have been shown to be suitable for calculating singlet-triplet energy differences. ${ }^{42,47-49}$ Here, excited-state calculations were performed using the EOM-CCSD/6-31G method implemented in the Q-Chem program package. ${ }^{50}$

Excitation energies, SOCs, vibronic couplings, transition-dipole moments, and permanent dipole moments were calculated using EOM-CCSD wave functions. At the TD-TPSSh level, $\mathrm{S}_{1}$ and $\mathrm{T}_{1}$ local-energy minima were located at the $\mathrm{S}_{1}$ and $\mathrm{T}_{1}$ optimized geometries, respectively. However, as shown in Supplementary Table 12 and Supplementary Fig. 3, the lowest $\mathrm{S}_{1}$ and $\mathrm{T}_{1}$ energy levels calculated at the EOM-CCSD level were both located at the $\mathrm{T}_{1}$ geometry (3.37 and $3.18 \mathrm{eV}$, respectively). In addition, the $\mathrm{S}_{1}$ and $\mathrm{T}_{1}$ structures were nearly the same including the dihedral angles (Supplementary Table 5); hence, the structure was denoted the $\mathrm{S}_{1} \mathrm{~T}_{1}$ geometry. Because the Stokes shift was experimentally observed for DABNA-1 in solution and in solid films ${ }^{10}$ it was reasonably assumed that the initial TADF state was the $\mathrm{S}_{1}$ adiabatic energy minimum. The $S_{0}, S_{1}, T_{1}$, and $T_{2}$ electronic states and the $S_{1} T_{1}$ and $T_{2}$ equilibrium geometries were used to model TADF for DABNA-1 (eight electronic states in all). Because $\mathrm{S}_{2}$ states were $0.89 \mathrm{eV}$ higher in energy than the $\mathrm{S}_{1}$ states, their contributions were neglected (Supplementary Fig. 3). The $T_{3}$ states were $0.2 \mathrm{eV}$ higher than $\mathrm{S}_{1}$, but were neglected because the electronictransition rate constants from $S_{1}$ to $T_{3}$ were far smaller than those of the competing processes $\left(S_{1}\right.$ 
to $T_{1}$ and $S_{1}$ to $T_{2}$ ) and that from $T_{2}$ to $T_{3}$ was smaller than that from $T_{2}$ to $T_{1}$ (Supplementary

Fig. 3). In the EOM-CCSD calculations, $\mathrm{S}_{1}$ and $\mathrm{T}_{1}$ involved predominantly $\mathrm{HOMO} \rightarrow \mathrm{LUMO}$ transitions, whereas $\mathrm{T}_{2}$ involved predominantly a linear combination of $\mathrm{HOMO} \rightarrow \mathrm{LUMO}+5$ and HOMO-1 $\rightarrow$ LUMO transitions (Fig. 1a, Supplementary Tables 6-9). Figure 1b shows a calculated energy-level diagram of DABNA-1. The adiabatic $\mathrm{T}_{1} \rightarrow \mathrm{S}_{1}$ energy difference calculated with the EOM-CCSD/6-31G(d) method $\left(\mathrm{S}_{1} \mathrm{~T}_{1}\right.$ geometry) was only $0.06 \mathrm{eV}$ larger than the experimentally obtained $0.2 \mathrm{eV}$. Thus, EOM-CCSD significantly improved the overestimation of $\Delta E\left(\mathrm{~T}_{1} \rightarrow \mathrm{S}_{1}\right)$, relative to those by previously reported TD-DFT methods. ${ }^{40,42-44}$

Calculation of rate constants and luminescence quantum efficiencies. We examined triplet formation from $\mathrm{S}_{1}$. Figure $1 \mathrm{~b}$ shows calculated energy differences and SOCs, and Table 1 lists calculated and experimental values of $k_{\mathrm{IC}}, k_{\mathrm{ISC}}, k_{\mathrm{RISC}}, k_{\mathrm{F}}$, and $k_{\mathrm{Phos}}$. The raw rate constants in Table 1 were calculated using excitation energies calculated via EOM-CCSD. (Corrected values are discussed below.) We evaluated the consistency between the raw and experimental rate constants. Equations for $k_{\mathrm{IC}}, k_{\mathrm{ISC}}, k_{\mathrm{RISC}}, k_{\mathrm{F}}$, and $k_{\mathrm{Phos}}$ are given in the Supplementary Information (Supplementary Equations S1-S9).

Calculated values of $k_{\mathrm{F}}\left(\mathrm{S}_{1} \rightarrow \mathrm{S}_{0}\right)\left(1.4 \times 10^{8} \mathrm{~s}^{-1}\right)$ and $k_{\mathrm{IC}}\left(\mathrm{S}_{1} \rightarrow \mathrm{S}_{0}\right)\left(1.2 \times 10^{7} \mathrm{~s}^{-1}\right)$ agreed quantitatively with experimental results $\left(1.0 \times 10^{8} \mathrm{~s}^{-1}\right.$ and $1.3 \times 10^{7} \mathrm{~s}^{-1}$, respectively) determined by Hatakeyama et al. ${ }^{10}$ from PL decay curves of a 1-wt\% DABNA-1: 9,9'-biphenyl-3,3'-diylbis$9 H$-carbazole film at $300 \mathrm{~K}$ (Table 1$)$. The experimentally obtained $k_{\text {ISC }}$ value $\left(4.5 \times 10^{6} \mathrm{~s}^{-1}\right)$ agreed with $k_{\mathrm{ISC}}\left(\mathrm{S}_{1} \rightarrow \mathrm{T}_{2}\right)\left(5.4 \times 10^{6} \mathrm{~s}^{-1}\right)$, rather than $k_{\mathrm{ISC}}\left(\mathrm{S}_{1} \rightarrow \mathrm{T}_{1}\right)\left(1.7 \times 10^{5} \mathrm{~s}^{-1}\right)$, suggesting that the experimental $k_{\mathrm{ISC}}$ should be assigned to $k_{\mathrm{ISC}}\left(\mathrm{S}_{1} \rightarrow \mathrm{T}_{2}\right)$. The $k_{\mathrm{ISC}}\left(\mathrm{S}_{1} \rightarrow \mathrm{T}_{2}\right)$ was ten times greater than $k_{\mathrm{ISC}}\left(\mathrm{S}_{1} \rightarrow \mathrm{T}_{1}\right)$, because of the larger $\mathrm{S}_{1}-\mathrm{T}_{2} \mathrm{SOC}\left(1.52 \mathrm{~cm}^{-1}\right)$ relative to that of $\mathrm{S}_{1}-\mathrm{T}_{1}\left(0.06 \mathrm{~cm}^{-1}\right)$. The large $\mathrm{S}_{1} \rightarrow \mathrm{T}_{2}$ SOC enhanced $\mathrm{S}_{1} \rightarrow \mathrm{T}_{2}$ ISC, despite the uphill transition from $\mathrm{S}_{1}$ to $\mathrm{T}_{2}$ (the 
Franck-Condon energy difference was $135 \mathrm{meV}$, Fig. 1b), compared with the downhill transition from $\mathrm{S}_{1}$ to $\mathrm{T}_{1}(260 \mathrm{meV})$. The small $\mathrm{S}_{1}-\mathrm{T}_{1} \mathrm{SOC}$ resulted from very similar $\mathrm{S}_{1}$ and $\mathrm{T}_{1}$ orbital configurations (HOMO $\rightarrow$ LUMO transition). These results suggested that the ISC $\left(\mathrm{S}_{1} \rightarrow \mathrm{T}_{1}\right.$ conversion) of DABNA-1 occurred via the stepwise $\mathrm{S}_{1} \rightarrow \mathrm{T}_{2} \rightarrow \mathrm{T}_{1}$ process, rather than by the onestep $\mathrm{S}_{1} \rightarrow \mathrm{T}_{1}$ ISC, irrespective of the geometry relaxation (gray arrows in Fig. 1c). Such $\mathrm{T}_{2}-$ supported ISC is experimentally observed for a TADF system when $\Delta E\left(\mathrm{~S}_{1} \rightarrow \mathrm{T}_{2}\right)$ is sufficiently small. ${ }^{51}$ Because $\mathrm{T}_{2} \rightarrow \mathrm{T}_{1}$ IC was much faster than $\mathrm{S}_{1} \rightarrow \mathrm{T}_{2}$ ISC, the latter was the rate determining step of the $\mathrm{S}_{1} \rightarrow \mathrm{T}_{2} \rightarrow \mathrm{T}_{1}$ process (Fig. 1c). Hence, the net rate constant $k\left(\mathrm{~S}_{1} \rightarrow \mathrm{T}_{2} \rightarrow \mathrm{T}_{1}\right)$ was approximately equal to $k_{\mathrm{ISC}}\left(\mathrm{S}_{1} \rightarrow \mathrm{T}_{2}\right)=5.4 \times 10^{6} \mathrm{~s}^{-1}$ (Table 1$)$.

For RISC from $\mathrm{T}_{1}$, the calculated $k_{\mathrm{RISC}}\left(\mathrm{T}_{1} \rightarrow \mathrm{S}_{1}\right)\left(2.5 \mathrm{~s}^{-1}\right)$ was considerably less than the experimental value $\left(1 \times 10^{4} \mathrm{~s}^{-1}\right),{ }^{10}$ indicating that $\mathrm{T}_{1} \rightarrow \mathrm{S}_{1}$ conversion did not occur in one-step but via a more effective process. Because the $\mathrm{S}_{2}$ and $\mathrm{T}_{3}$ energy levels, as well as higher singlet and triplet states, could not contribute to the RISC from $\mathrm{T}_{1}$, as discussed above, and $\mathrm{T}_{2}$ was close to $\mathrm{S}_{1}$ (see Supplementary Fig. 3), stepwise $\mathrm{T}_{1} \rightarrow \mathrm{T}_{2} \rightarrow \mathrm{S}_{1}$ was the most promising mechanism (green arrows in Fig. 1c). To determine $k\left(\mathrm{~T}_{1} \rightarrow \mathrm{T}_{2} \rightarrow \mathrm{S}_{1}\right)$, we calculated the trPL decay curve (Fig. 2) via kinetic equations (Supplementary Equations S10 - S17 and Supplementary Table 15). The total PLQY $(\Phi)$ and the TADF lifetime ( $\left.\tau_{\mathrm{TADF}}\right)$ were then obtained from the trPL decay curve (Fig. 2 ). The prompt and delayed components of $\Phi\left(\Phi_{\mathrm{P}}\right.$ and $\Phi_{\mathrm{TADF}}$, respectively) were calculated from:

$$
\begin{gathered}
\Phi_{\mathrm{P}}=\frac{k_{\mathrm{F}}\left(\mathrm{S}_{1} \rightarrow \mathrm{S}_{0}\right)}{k_{\mathrm{F}}\left(\mathrm{S}_{1} \rightarrow \mathrm{S}_{0}\right)+k_{\mathrm{IC}}\left(\mathrm{S}_{1} \rightarrow \mathrm{S}_{0}\right)+k_{\mathrm{ISC}}\left(\mathrm{S}_{1} \rightarrow \mathrm{T}_{2}\right)+k_{\mathrm{ISC}}\left(\mathrm{S}_{1} \rightarrow \mathrm{T}_{1}\right)} \\
\Phi_{\mathrm{TADF}}=\Phi-\Phi_{\mathrm{P}}
\end{gathered}
$$

and the ISC quantum yield $\left(\Phi_{\text {ISC }}\right)$ was calculated from:

$$
\Phi_{\mathrm{ISC}}=\frac{k_{\mathrm{ISC}}\left(\mathrm{S}_{1} \rightarrow \mathrm{T}_{2}\right)+k_{\mathrm{ISC}}\left(\mathrm{S}_{1} \rightarrow \mathrm{T}_{1}\right)}{k_{\mathrm{F}}\left(\mathrm{S}_{1} \rightarrow \mathrm{S}_{0}\right)+k_{\mathrm{IC}}\left(\mathrm{S}_{1} \rightarrow \mathrm{S}_{0}\right)+k_{\mathrm{ISC}}\left(\mathrm{S}_{1} \rightarrow \mathrm{T}_{2}\right)+k_{\mathrm{ISC}}\left(\mathrm{S}_{1} \rightarrow \mathrm{T}_{1}\right)}
$$


Then, $k_{\mathrm{TADF}}$ was determined from: ${ }^{10}$

$$
k_{\mathrm{TADF}}=\frac{\Phi_{\mathrm{TADF}}}{\Phi_{\mathrm{ISC}} \tau_{\mathrm{TADF}}}
$$

Finally, $k\left(\mathrm{~T}_{1} \rightarrow \mathrm{T}_{2} \rightarrow \mathrm{S}_{1}\right)$ was calculated from:

$$
k\left(\mathrm{~T}_{1} \rightarrow \mathrm{T}_{2} \rightarrow \mathrm{S}_{1}\right)=\frac{k_{\mathrm{F}}\left(\mathrm{S}_{1} \rightarrow \mathrm{S}_{0}\right) \times k_{\mathrm{TADF}}}{k_{\mathrm{F}}\left(\mathrm{S}_{1} \rightarrow \mathrm{S}_{0}\right)-k\left(\mathrm{~S}_{1} \rightarrow \mathrm{T}_{2} \rightarrow \mathrm{T}_{1}\right)}
$$

Table 1 also lists calculated $\Phi, \Phi_{\mathrm{P}}, \Phi_{\mathrm{TADF}}, \Phi_{\mathrm{ISC}}, \tau_{\mathrm{TADF}}, k_{\mathrm{TADF}}, k\left(\mathrm{~T}_{1} \rightarrow \mathrm{T}_{2} \rightarrow \mathrm{S}_{1}\right)$, and $k\left(\mathrm{~S}_{1} \rightarrow \mathrm{T}_{2} \rightarrow \mathrm{T}_{1}\right)$ values. The $\Phi, \Phi_{\mathrm{P}}, \Phi_{\mathrm{TADF}}$, and $\Phi_{\mathrm{ISC}}$ values were quantitatively consistent with the experimental values because of the quantitative predictions for $k_{\mathrm{F}}\left(\mathrm{S}_{1} \rightarrow \mathrm{S}_{0}\right), k_{\mathrm{IC}}\left(\mathrm{S}_{1} \rightarrow \mathrm{S}_{0}\right), k_{\mathrm{ISC}}\left(\mathrm{S}_{1} \rightarrow \mathrm{T}_{2}\right)$, and $k_{\mathrm{ISC}}\left(\mathrm{S}_{1} \rightarrow \mathrm{T}_{1}\right)$. The calculated conversion rate constant $k\left(\mathrm{~S}_{1} \rightarrow \mathrm{T}_{2} \rightarrow \mathrm{T}_{1}\right)$ was consistent with the experimental $k_{\mathrm{ISC}}$, suggesting that the experimental ISC should be assigned to $\mathrm{S}_{1} \rightarrow \mathrm{T}_{2} \rightarrow \mathrm{T}_{1}$ ISC rather than direct $\mathrm{S}_{1} \rightarrow \mathrm{T}_{1}$ ISC. All the calculated single step rate constants and $k\left(\mathrm{~S}_{1} \rightarrow \mathrm{T}_{2} \rightarrow \mathrm{T}_{1}\right)$ agreed well with the experimental values. However, the agreements of the calculated $k_{\text {TADF }}$ and $k\left(\mathrm{~T}_{1} \rightarrow \mathrm{T}_{2} \rightarrow \mathrm{S}_{1}\right)\left(0.16 \times 10^{4} \mathrm{~s}^{-1}\right.$ and $0.17 \times 10^{4} \mathrm{~s}^{-1}$, respectively $)$ and the experiments $\left(0.94 \times 10^{4} \mathrm{~s}^{-1}\right.$ and $1.0 \times 10^{4} \mathrm{~s}^{-1}$, respectively) were not as good as we expected, compared to the case of $k\left(\mathrm{~S}_{1} \rightarrow \mathrm{T}_{2} \rightarrow \mathrm{T}_{1}\right)$. Considering that they included the uphill-energy $\mathrm{T}_{1} \rightarrow \mathrm{T}_{2} \mathrm{IC}$, there was a possibility that $\Delta E\left(\mathrm{~T}_{1} \rightarrow \mathrm{T}_{2}\right)$ was overestimated, leading to an underestimation of $k_{\mathrm{IC}}\left(\mathrm{T}_{1} \rightarrow \mathrm{T}_{2}\right)$, $k_{\mathrm{TADF}}$, and $k\left(\mathrm{~T}_{1} \rightarrow \mathrm{T}_{2} \rightarrow \mathrm{S}_{1}\right)$.

To examine the effect of $\Delta E\left(\mathrm{~T}_{1} \rightarrow \mathrm{T}_{2}\right)$ on $k_{\mathrm{IC}}\left(\mathrm{T}_{1} \rightarrow \mathrm{T}_{2}\right), k_{\mathrm{TADF}}$, and $k\left(\mathrm{~T}_{1} \rightarrow \mathrm{T}_{2} \rightarrow \mathrm{S}_{1}\right)$, we recalculated these rate constants using a corrected $\Delta E\left(\mathrm{~T}_{1} \rightarrow \mathrm{T}_{2}\right)$. The rate-determining step for the $\mathrm{T}_{2}$-mediated RISC was $\mathrm{T}_{1} \rightarrow \mathrm{T}_{2}$ IC; hence, $\Delta E\left(\mathrm{~T}_{1} \rightarrow \mathrm{T}_{2}\right)$ could be viewed as the activation energy for DABNA-1 TADF and RISC. As discussed above, the energy difference between the calculated and experimental $\mathrm{T}_{1} \rightarrow \mathrm{S}_{1}$ was $60 \mathrm{meV}$. Thus, $60 \mathrm{meV}$ was subtracted from the EOMCCSD-calculated $\Delta E\left(\mathrm{~T}_{1} \rightarrow \mathrm{T}_{2}\right)$ value of $252 \mathrm{meV}$. The energy-levels and rate constants calculated 
with the corrected $\Delta E\left(\mathrm{~T}_{1} \rightarrow \mathrm{T}_{2}\right)$ are also listed in Table 1 . The value of $k\left(\mathrm{~S}_{1} \rightarrow \mathrm{T}_{2} \rightarrow \mathrm{T}_{1}\right)$ was unchanged, suggesting that the $\mathrm{T}_{1}$ energy correction had little effect on the ISC. In contrast, $k_{\mathrm{IC}}\left(\mathrm{T}_{1} \rightarrow \mathrm{T}_{2}\right)$ increased from $1.1 \times 10^{7} \mathrm{~s}^{-1}$ to $3.3 \times 10^{8} \mathrm{~s}^{-1}$. As a result, $k_{\mathrm{TADF}}$ and $k\left(\mathrm{~T}_{1} \rightarrow \mathrm{T}_{2} \rightarrow \mathrm{S}_{1}\right)$ increased to $1.8 \times 10^{4} \mathrm{~s}^{-1}$ and $1.9 \times 10^{4} \mathrm{~s}^{-1}$, respectively, which were in much better agreements with the experiments. These results suggested that the $\Delta E\left(\mathrm{~T}_{1} \rightarrow \mathrm{T}_{2}\right)$ overestimation was responsible for the underestimation of $k_{\mathrm{TADF}}$ and $k\left(\mathrm{~T}_{1} \rightarrow \mathrm{T}_{2} \rightarrow \mathrm{S}_{1}\right)$. Thus, $k_{\mathrm{TADF}}$ and $k\left(\mathrm{~T}_{1} \rightarrow \mathrm{T}_{2} \rightarrow \mathrm{S}_{1}\right)$ depended largely on $k_{\mathrm{IC}}\left(\mathrm{T}_{1} \rightarrow \mathrm{T}_{2}\right)$, which was the sum of IC rate constants for individual molecular vibrations, $k_{\mathrm{IC}, \alpha}\left(\mathrm{T}_{1} \rightarrow \mathrm{T}_{2}\right)$, where $\alpha$ denoted the $\alpha$ th vibrational mode (Supplementary Equations S5-S7). A combined in-plane and out-of-plane bending mode $\left(743 \mathrm{~cm}^{-1}\right)$ had the largest $k_{\mathrm{IC}, \alpha}\left(\mathrm{T}_{1} \rightarrow \mathrm{T}_{2}\right)$ value (Supplementary Table 14 and Supplementary Fig. 5), suggesting that the $\mathrm{T}_{1} \rightarrow \mathrm{T}_{2} \mathrm{IC}$ was predominantly accelerated by the bending mode.

\section{Discussion}

In this work, we calculated the IC, ISC, RISC, fluorescence, phosphorescence, and TADF rate constants for DABNA-1, using EOM-CCSD wave functions and Fermi's golden rule. The values quantitatively reproduced those from experiments, and thus validated the calculations. Various quantum yields, including PLQY, were also predicted and we revealed the DABNA-1 decay mechanism. The calculated population kinetics and the trPL decay curve indicated that TADF from DABNA-1 occurs via consecutive two processes, $\mathrm{T}_{1} \rightarrow \mathrm{T}_{2} \mathrm{IC}$ and $\mathrm{T}_{2} \rightarrow \mathrm{S}_{1} \mathrm{RISC}$. Our proposed method here will be useful for accurate prediction of all rate constants and quantum yields relevant to OLED phenomena for various compounds with a wide range of structures in future. 


\section{a}<smiles>c1ccc(N2c3ccccc3B3c4ccccc4N(c4ccccc4)c4cccc2c43)cc1</smiles>

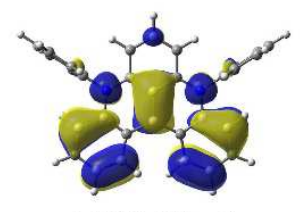

HOMO-1

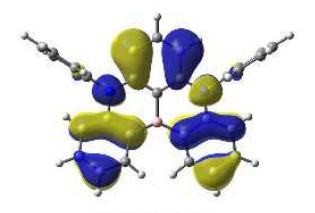

HOMO

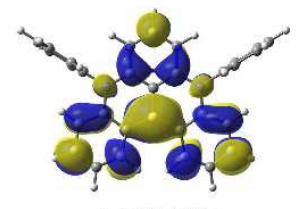

LUMO

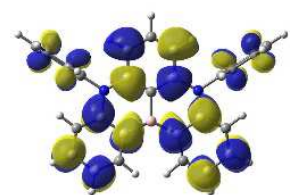

LUMO+5 b $\mathrm{S}_{1} \mathrm{~T}_{1}$-geom

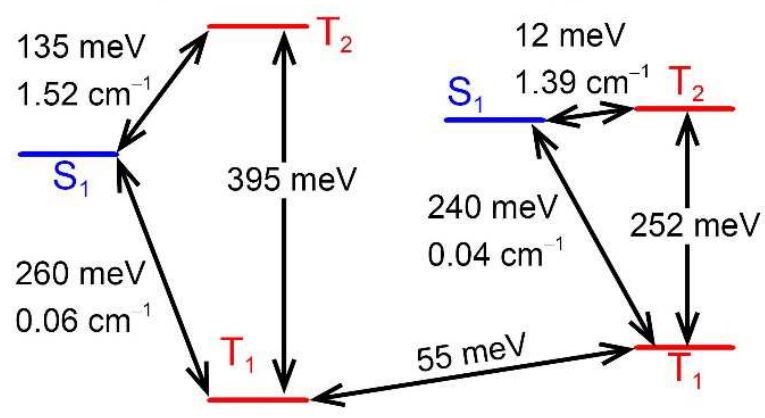

d $\mathrm{S}_{1} \mathrm{~T}_{1}$-geom

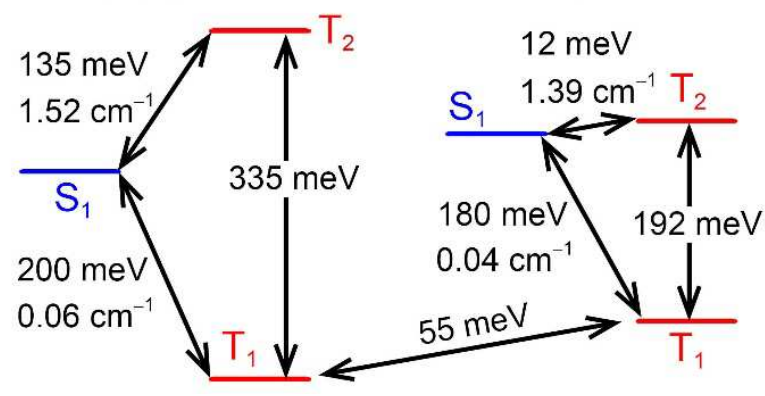

C $S_{1} T_{1}$-geom

$\mathrm{T}_{2}$-geom

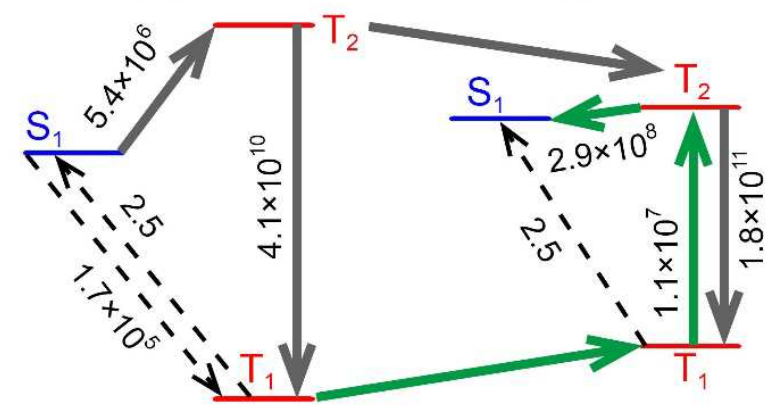

e $\mathrm{S}_{1} \mathrm{~T}_{1}$-geom $\quad \mathrm{T}_{2}$-geom

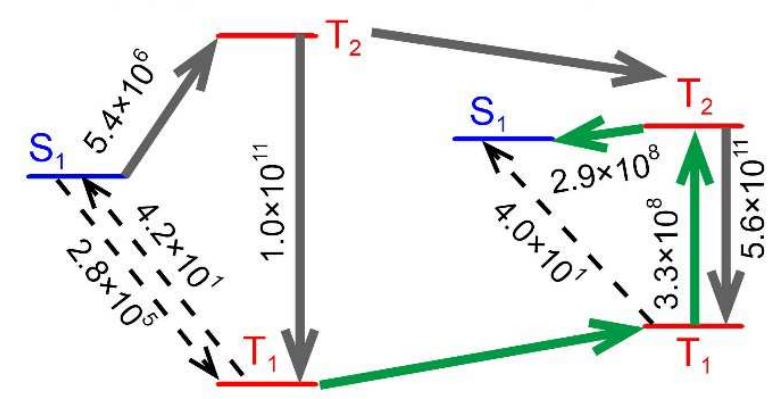

Fig. 1 Molecular orbitals and electronic transitions of DABNA-1. a Structure of DABNA-1

and HOMO-1, HOMO, LUMO, and LUMO+5 distributions calculated at the RHF/6-31G level $(\mathrm{HOMO}=$ highest-occupied molecular orbital, LUMO = lowest-unoccupied molecular orbital $) . \mathbf{b}$ Energy differences (meV) between $\mathrm{S}_{1}, \mathrm{~T}_{1}$, and $\mathrm{T}_{2} . \mathrm{S}_{1}-\mathrm{T}_{1}$ and $\mathrm{S}_{1}-\mathrm{T}_{2}$ spin-orbit couplings $\left(\mathrm{cm}^{-1}\right)$. c The stepwise $\mathrm{S}_{1} \rightarrow \mathrm{T}_{2} \rightarrow \mathrm{T}_{1}$ and $\mathrm{T}_{1} \rightarrow \mathrm{T}_{2} \rightarrow \mathrm{S}_{1}$ processes of DABNA-1. The grey solid arrow depicts stepwise $\mathrm{S}_{1} \rightarrow \mathrm{T}_{2} \rightarrow \mathrm{T}_{1}$; the green solid arrow depicts stepwise $\mathrm{T}_{1} \rightarrow \mathrm{T}_{2} \rightarrow \mathrm{S}_{1}$; the dotted arrows show minor decay and up-conversion processes. The values are calculated rate constants for one- 
step transitions. d The energy differences calculated using the corrected $\mathrm{T}_{1}$ energy. e Calculated rate constants for one-step transitions using the corrected $\mathrm{T}_{1}$ energy.

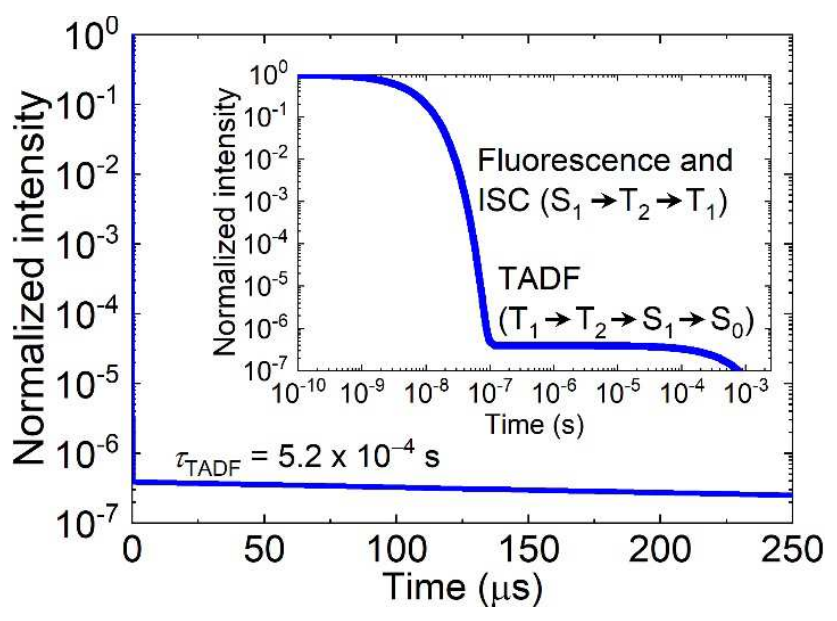

Fig. 2 Calculated transient photoluminescence (trPL) decay curve. The inset is a log-log plot of the decay curve. 


\section{References}

1 Uoyama, H., Goushi, K., Shizu, K., Nomura, H. \& Adachi, C. Highly efficient organic light-emitting diodes from delayed fluorescence. Nature 492, 234-238 (2012).

2 Adachi, C. Third-generation organic electroluminescence materials. Jpn. J. Appl. Phys. 53, 060101 (2014).

3 Wong, M. Y. \& Zysman-Colman, E. Purely Organic Thermally Activated Delayed Fluorescence Materials for Organic Light-Emitting Diodes. Adv. Mater. 29, 1605444 (2017). 4 Tanaka, H., Shizu, K., Miyazaki, H. \& Adachi, C. Efficient green thermally activated delayed fluorescence (TADF) from a phenoxazine-triphenyltriazine (PXZ-TRZ) derivative. Chem. Commun. 48, 11392-11394 (2012).

5 Zhang, Q. et al. Design of Efficient Thermally Activated Delayed Fluorescence Materials for Pure Blue Organic Light Emitting Diodes. J. Am. Chem. Soc. 134, 14706-14709 (2012). $6 \quad$ Kaji, H. et al. Purely organic electroluminescent material realizing $100 \%$ conversion from electricity to light. Nat. Commun. 6, 8476 (2015).

7 Shizu, K. et al. Strategy for Designing Electron Donors for Thermally Activated Delayed Fluorescence Emitters. J. Phys. Chem. C 119, 1291-1297 (2015).

8 Shizu, K. et al. Enhanced Electroluminescence from a Thermally Activated DelayedFluorescence Emitter by Suppressing Nonradiative Decay. Phys. Rev. Applied 3, 014001 (2015).

9 Wada, Y., Nakagawa, H., Matsumoto, S., Wakisaka, Y. \& Kaji, H. Organic light emitters exhibiting very fast reverse intersystem crossing. Nat. Photon. 14, 643-649 (2020).

10 Hatakeyama, T. et al. Ultrapure Blue Thermally Activated Delayed Fluorescence Molecules: Efficient HOMO-LUMO Separation by the Multiple Resonance Effect. Adv. Mater. 28, 2777-2781 (2016).

11 Nakatsuka, S., Gotoh, H., Kinoshita, K., Yasuda, N. \& Hatakeyama, T. Divergent Synthesis of Heteroatom-Centered 4,8,12-Triazatriangulenes. Angew. Chem. Int. Ed. 56, 50875090 (2017).

12 Matsui, K. et al. One-Shot Multiple Borylation toward BN-Doped Nanographenes. J. Am. Chem. Soc. 140, 1195-1198 (2018).

13 Liang, X. et al. Peripheral Amplification of Multi-Resonance Induced Thermally Activated Delayed Fluorescence for Highly Efficient OLEDs. Angew. Chem. Int. Ed. 57, 1131611320 (2018).

14 Han, S. H., Jeong, J. H., Yoo, J. W. \& Lee, J. Y. Ideal blue thermally activated delayed fluorescence emission assisted by a thermally activated delayed fluorescence assistant dopant through a fast reverse intersystem crossing mediated cascade energy transfer process. J. Mater. Chem. C 7, 3082-3089 (2019).

15 Kondo, Y. et al. Narrowband deep-blue organic light-emitting diode featuring an organoboron-based emitter. Nat. Photon. 13, 678-682 (2019).

16 Oda, S., Kawakami, B., Kawasumi, R., Okita, R. \& Hatakeyama, T. Multiple Resonance Effect-Induced Sky-Blue Thermally Activated Delayed Fluorescence with a Narrow Emission Band. Org. Lett. 21, 9311-9314 (2019).

17 Zhang, Y. et al. Multi-Resonance Induced Thermally Activated Delayed Fluorophores for Narrowband Green OLEDs. Angew. Chem. Int. Ed. 58, 16912-16917 (2019).

18 Suresh, S. M. et al. A Deep Blue B,N-Doped Heptacene Emitter That Shows Both Thermally Activated Delayed Fluorescence and Delayed Fluorescence by Triplet-Triplet Annihilation. J. Am. Chem. Soc. 142, 6588-6599 (2020). 
19 Zhang, Y. et al. Achieving Pure Green Electroluminescence with CIEy of 0.69 and EQE of 28.2\% from an Aza-Fused Multi-Resonance Emitter. Angew. Chem. Int. Ed. 59, 17499-17503 (2020).

20 Yang, M., Park, I. S. \& Yasuda, T. Full-Color, Narrowband, and High-Efficiency

Electroluminescence from Boron and Carbazole Embedded Polycyclic Heteroaromatics. J. Am. Chem. Soc. 142, 19468-19472 (2020).

21 Madayanad Suresh, S., Hall, D., Beljonne, D., Olivier, Y. \& Zysman-Colman, E.

Multiresonant Thermally Activated Delayed Fluorescence Emitters Based on Heteroatom-Doped Nanographenes: Recent Advances and Prospects for Organic Light-Emitting Diodes. Adv. Func. Mater. 30, 1908677 (2020).

22 Tanaka, H. et al. Hypsochromic Shift of Multiple-Resonance-Induced Thermally Activated Delayed Fluorescence by Oxygen Atom Incorporation. Angew. Chem. Int. Ed. 60, 17910-17914 (2021).

23 Oda, S. et al. Carbazole-Based DABNA Analogues as Highly Efficient Thermally Activated Delayed Fluorescence Materials for Narrowband Organic Light-Emitting Diodes. Angew. Chem. Int. Ed. 60, 2882-2886 (2021).

24 Nagata, M. et al. Fused-Nonacyclic Multi-Resonance Delayed Fluorescence Emitter Based on Ladder-Thiaborin Exhibiting Narrowband Sky-Blue Emission with Accelerated Reverse Intersystem Crossing. Angew. Chem. Int. Ed. 60, 20280-20285 (2021).

25 Yang, M. et al. Wide-Range Color Tuning of Narrowband Emission in Multi-resonance Organoboron Delayed Fluorescence Materials through Rational Imine/Amine Functionalization. Angew. Chem. Int. Ed. 60, 23142-23147 (2021).

26 Yuan, Y. et al. The Design of Fused Amine/Carbonyl System for Efficient Thermally Activated Delayed Fluorescence: Novel Multiple Resonance Core and Electron Acceptor. Adv. Opt. Mater. 7, 1801536 (2019).

27 Hall, D. et al. Improving Processability and Efficiency of Resonant TADF Emitters: A Design Strategy. Adv. Opt. Mater. 8, 1901627 (2020).

28 Naveen, K. R. et al. Achieving High Efficiency and Pure Blue Color in Hyperfluorescence Organic Light Emitting Diodes using Organo-Boron Based Emitters. $A d v$. Func. Mater. 2110356 (2021).

29 Ikeda, N. et al. Solution-Processable Pure Green Thermally Activated Delayed Fluorescence Emitter Based on the Multiple Resonance Effect. Adv. Mater. 32, 2004072 (2020). 30 Sun, D. et al. The design of an extended multiple resonance TADF emitter based on a polycyclic amine/carbonyl system. Mater. Chem. Front. 4, 2018-2022 (2020).

31 Liu, G. et al. Facile synthesis of multi-resonance ultra-pure-green TADF emitters based on bridged diarylamine derivatives for efficient OLEDs with narrow emission. J. Mater. Chem. C 9, 8308-8313 (2021).

32 Hua, T. et al. Heavy-atom effect promotes multi-resonance thermally activated delayed fluorescence. Chem. Eng. J. 426, 131169 (2021).

33 Jiang, P. et al. Simple Acridan-Based Multi-Resonance Structures Enable Highly Efficient Narrowband Green TADF Electroluminescence. Adv. Opt. Mater. 9, 2100825 (2021). 34 Jiang, P. et al. Quenching-Resistant Multiresonance TADF Emitter Realizes 40\% External Quantum Efficiency in Narrowband Electroluminescence at High Doping Level. Adv. Mater. 34, 2106954 (2021). 
$35 \mathrm{Wu}, \mathrm{X}$. et al. Fabrication of Circularly Polarized MR-TADF Emitters with Asymmetrical Peripheral-Lock Enhancing Helical B/N-Doped Nanographenes. Adv. Mater. 34, 2105080 (2022).

36 Xu, Y., Wang, Q., Cai, X., Li, C. \& Wang, Y. Highly Efficient Electroluminescence from Narrowband Green Circularly Polarized Multiple Resonance Thermally Activated Delayed Fluorescence Enantiomers. Adv. Mater. 33, 2100652 (2021).

$37 \mathrm{Xu}, \mathrm{Y}$. et al. Materials with High Color Purity Based on Strong Acceptor Attachment onto B-N-Containing Multiple Resonance Frameworks. CCS Chem 3, 2077-2091 (2021). 38 Liu, Y., Xiao, X., Ran, Y., Bin, Z. \& You, J. Molecular design of thermally activated delayed fluorescent emitters for narrowband orange-red OLEDs boosted by a cyanofunctionalization strategy. Chem. Sci. 12, 9408-9412 (2021).

39 Zhang, Y. et al. Multi-Resonance Deep-Red Emitters with Shallow Potential-Energy Surfaces to Surpass Energy-Gap Law. Angew. Chem. Int. Ed. 60, 20498-20503 (2021).

40 Northey, T. \& Penfold, T. J. The intersystem crossing mechanism of an ultrapure blue organoboron emitter. Org. Electron. 59, 45-48 (2018).

41 Gao, Y. et al. Realizing performance improvement of blue thermally activated delayed fluorescence molecule DABNA by introducing substituents on the para-position of boron atom. Chem. Phys. Lett. 701, 98-102 (2018).

42 Pershin, A. et al. Highly emissive excitons with reduced exchange energy in thermally activated delayed fluorescent molecules. Nat. Commun. 10, 597 (2019).

43 Hall, D. et al. The modelling of multi-resonant thermally activated delayed fluorescence emitters - properly accounting for electron correlation is key! Preprint at https://chemrxiv.org/engage/chemrxiv/article-details/6173bad70c048095df40e5f3 (2021).

44 Lin, L., Fan, J., Cai, L. \& Wang, C.-K. Excited state dynamics of new-type thermally activated delayed fluorescence emitters: theoretical view of light-emitting mechanism. Mol. Phys. 116, 19-28 (2018).

45 Shizu, K. \& Kaji, H. Theoretical Determination of Rate Constants from Excited States: Application to Benzophenone. J. Phys. Chem. A 125, 9000-9010 (2021).

46 Frisch, M. J. et al. Gaussian 16 Rev. C.01. (2016).

47 de Silva, P. Inverted Singlet-Triplet Gaps and Their Relevance to Thermally Activated Delayed Fluorescence. J. Phys. Chem. Lett. 10, 5674-5679 (2019).

48 Pios, S., Huang, X., Sobolewski, A. L. \& Domcke, W. Triangular boron carbon nitrides: an unexplored family of chromophores with unique properties for photocatalysis and optoelectronics. Phys. Chem. Chem. Phys. 23, 12968-12975 (2021).

49 Ehrmaier, J. et al. Singlet-Triplet Inversion in Heptazine and in Polymeric Carbon Nitrides. J. Phys. Chem. A 123, 8099-8108 (2019).

50 Shao, Y. et al. Advances in molecular quantum chemistry contained in the Q-Chem 4 program package. Mol. Phys. 113, 184-215 (2015).

51 Han, C. et al. Ladder-like energy-relaying exciplex enables $100 \%$ internal quantum efficiency of white TADF-based diodes in a single emissive layer. Nat. Commun. 12, 3640 (2021). 


\section{Acknowledgments}

The quantum chemical calculations using the Gaussian 16 and Q-Chem program packages were performed on the SuperComputer System, Institute for Chemical Research, Kyoto University. It was also supported by JSPS KAKENHI grant numbers: 19K05629 and JP20H05840 (Grant-inAid for Transformative Research Areas, “Dynamic Exciton”). We thank Edanz (https://jp.edanz.com/ac) for editing a draft of this manuscript.

\section{Author Contributions}

K.S. performed the theoretical calculations. H.K. planned and supervised the project. All authors contributed to the writing of this manuscript and have approved the final version.

\section{Competing Interests}

The authors have no competing interests.

\section{Additional information}

Supplementary Information The online version contains supplementary material available at https://*** 


\section{Figure Legends and Tables}

Fig. 1 Molecular orbitals and electronic transitions of DABNA-1. a Structure of DABNA-1 and HOMO-1, HOMO, LUMO, and LUMO+5 distributions calculated at the RHF/6-31G level (HOMO = highest-occupied molecular orbital, LUMO = lowest-unoccupied molecular orbital). b Energy differences $(\mathrm{meV})$ between $\mathrm{S}_{1}, \mathrm{~T}_{1}$, and $\mathrm{T}_{2} . \mathrm{S}_{1}-\mathrm{T}_{1}$ and $\mathrm{S}_{1}-\mathrm{T}_{2}$ spin-orbit couplings $\left(\mathrm{cm}^{-1}\right)$. c The stepwise $\mathrm{S}_{1} \rightarrow \mathrm{T}_{2} \rightarrow \mathrm{T}_{1}$ and $\mathrm{T}_{1} \rightarrow \mathrm{T}_{2} \rightarrow \mathrm{S}_{1}$ processes of DABNA-1. The grey solid arrow depicts stepwise $\mathrm{S}_{1} \rightarrow \mathrm{T}_{2} \rightarrow \mathrm{T}_{1}$; the green solid arrow depicts stepwise $\mathrm{T}_{1} \rightarrow \mathrm{T}_{2} \rightarrow \mathrm{S}_{1}$; the dotted arrows show

minor decay and up-conversion processes. The values are calculated rate constants for one-step transitions. d The energy differences calculated using the corrected $\mathrm{T}_{1}$ energy. e Calculated rate constants for one-step transitions using the corrected $\mathrm{T}_{1}$ energy.

Fig. 2 Calculated transient photoluminescence (trPL) decay curve. The inset is a log-log plot of the decay curve. 
Table 1 Calculated and experimental photophysical properties of DABNA-1. Experimental values are from Hatakeyama et al. ${ }^{10}$

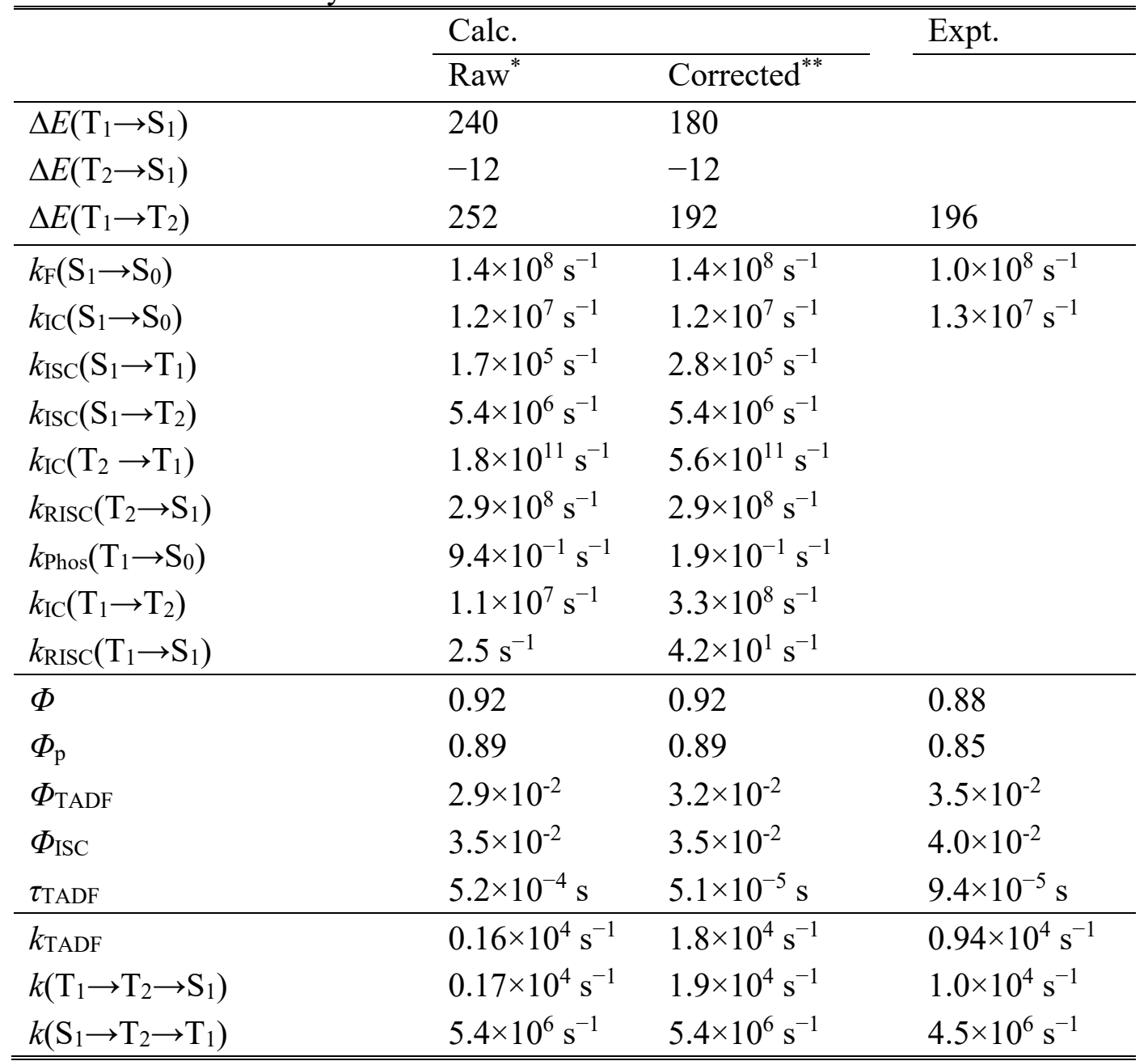

*) Energy differences were calculated using the EOM-CCSD/6-31G(d) method (Fig. 1b,c). ${ }^{* *}$ ) Corrected energy differences were used (Fig. 1d,e). 


\section{Supplementary Files}

This is a list of supplementary files associated with this preprint. Click to download.

- 20220215NatCommunSI.pdf 